\title{
NUMEROS CROMOSOMICOS DE CUATRO ESPECIES DE COSTUS (COSTACEAE), UNA DE CALATHEA, UNA DE MARANTA Y UNA DE STROMANTHE (MARANTACEAE)
}

\author{
ANDREW P. Vovides \\ Y \\ Maite Lascurain \\ Instituto de Ecología, A.C. \\ Apartado Postal 63 \\ 91000 Xalapa, Veracruz
}

\begin{abstract}
RESUMEN
Se hicieron recuentos cromosómicos de las siguientes especies: Costus pictus, C. scaber y C. pulverulentus tienen todos $2 n=18$; Costus dirzoi $2 n=27,28$; Calathea ovandensis $2 n=25$; Maranta gibba $2 n=40$ y Stromanthe macrochlamys $2 n=$ ca. 60 , ca. 63. Nuestras observaciones sobre Costus hacen pensar en la probable existencia de una serie poliploide, siendo triploide la recién descrita $C$. dirzoi.
\end{abstract}

\section{ABSTRACT}

Chromosome counts of the following species were done: Costus pictus, C. scaber and $C$. pulverulentus all have $2 n=18$; Costus dirzoi $2 n=27$, 28; Calathea ovandensis $2 n=25$; Maranta gibba $2 n=40$ and Stromanthe macrochlamys $2 n=$ ca. 60 , ca. 63. Observations on Costus lead us to consider the probable existence of a polyploid series, with the recently described $C$. dirzoi being triploid.

\section{INTRODUCCION}

Existen cuatro géneros y alrededor de 150 especies pantropicales en la familia Costaceae. El género más grande es Costus, con unas 100 especies; en México está representado por seis, distribuidas principalmente en el sureste de la República (Kress, 1990; Maas, 1972; Vovides, 1993, 1994). Se conocen 32 géneros y 525 especies de la familia Marantaceae a nivel mundial (Kennedy, com. pers.), la mayoría de América tropical (Heywood, 1993). De México se citan 5 géneros con alrededor de 15 especies. Hasta la fecha existen pocos trabajos citotaxonómicos de ambas familias (Andersson, 1981).

Los números cromosómicos somáticos previamente registrados para especies del género Costus son: $2 n=16$, 18, 36, 27 y 102 (Boem, 1931; Maas, 1972; Mahanty, 1970; Sato, 1960); para Calathea: $2 n=8,16,18,22,24,26,27$ y 52 (Mahanty, 1970; Sato, 1948, 1960); para Maranta: $2 n=8,12,18,24,26,28,32,33,48$ y 52 (Moore, 1973; Sato, 1960) y para Stromanthe: $2 n=18,22,36$ y 48 (Sato, 1960). 
De acuerdo con Raven (1975), el número básico $x=7$ es común a las familias de los órdenes o subórdenes Alismatales, Poales y Zingiberales. Esta información coincide con la observada para la clase Magnoliiflorae, considerada como primitiva y que presenta el número básico ancestral de las angiospermas (Dahlgren et al., 1985). Dentro del orden Zingiberales, existe una variación en el número cromosómico básico ya que se ha establecido $x=4,7,8,9,11,12,14,16,17$ y 26 (Dahlgren et al., 1985). El significado de la variación cromosómica fue discutido por Mahanty (1970) y Sato (1960).

El pequeño tamaño de los cromosomas y la variación en el número podría deberse a la existencia de cromosomas con satélites (constricciones secundarias) o cromosomas $B$, los cuales son difíciles de distinguir de los ordinarios (A) (Andersson, com. pers.).

En el presente trabajo se presentan por primera vez los números cromosómicos de: 1.- Calathea ovandensis Matuda; 2.- Costus dirzoi García-Mend. \& Ibarra-Manríquez; 3.Maranta gibba Sm. y 4.- Stromanthe macrochlamys (Wood \& Standl.) Kennedy \& Nicolson. Asimismo se obtuvieron los números cromosómicos diploides para: 5.- Costus scaber Ruiz \& Pavón; 6.- Costus pictus D. Don, y 7.- Costus pulverulentus Presl, de los cuales existen registros previos (Cuadro 2). La distribución de las especies estudiadas se presenta en la figura 1.

\section{METODOLOGIA}

Se obtuvieron ejemplares vivos de las distintas especies a estudiar en diversas localidades de los estados de Veracruz y Tabasco, mismos que fueron establecidos y cultivados en el Jardín Botánico Fco. J. Clavijero ubicado cerca de Xalapa, Veracruz. Los números de acceso de los ejemplares y números de colector se enlistan en el Cuadro 1. El material de respaldo se encuentra depositado en el herbario XAL.

Cuadro 1. Individuos usados para conteos enlistados por su número de acceso al Jardín Botánico y número de colecta, depositados en (XAL).

\begin{tabular}{lcl}
\hline ESPECIE & NUM. DE ACCESO & NUM. DE COLECTOR \\
\hline Calathea ovandensis & $92-225$ & H. O. Rivera 102 \\
Costus dirzoi & $82-277$ & G. Pattison 217 \\
C. pictus & $84-056$ & M. Nee s/n \\
C. pulverulentus & $85-049$ & C. Delfín 19 \\
C. scaber & $82-279$ & G. Pattison 219 \\
Maranta gibba & $92-226$ & M. Lascurain 200 \\
Stromanthe macrochlamys & $92-227$ & H. O. Rivera 1025 \\
\hline
\end{tabular}

Las localidades de colecta de los ejemplares revisados son:

1. Calathea ovandensis Matuda, procedente de La Maquinaria, municipio Amatlán de los Reyes, Ver., localizada en vegetación secundaria de selva mediana subcaducifolia. 
Vovides y Lascurain: Números Cromosómicos de Costus, Calathea, Maranta y Stromanthe

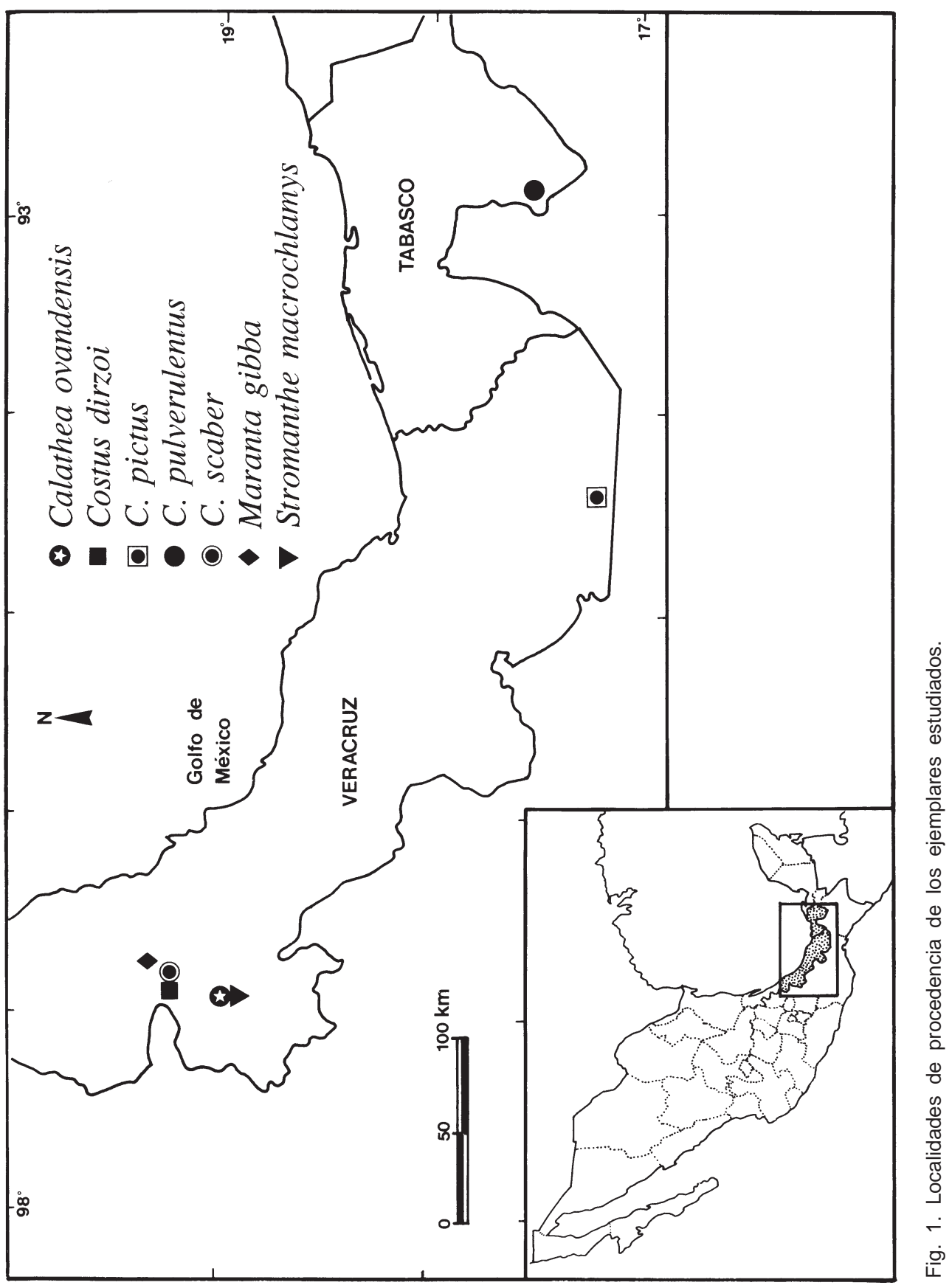


2. Costus dirzoi García-Mend. \& Ibarra-Manríquez, originaria de la Barranca del Coyotito, municipio Tenampa, Ver., en vegetación transicional entre bosque mesófilo de montaña y selva alta perennifolia.

3. Maranta gibba Sm., proveniente de la barranca de Acapan, del municipio de Jalcomulco, Ver., en vegetación riparia adyacente a un cultivo de mango.

4. Stromanthe macrochlamys (Wood \& Standl.) Kennedy \& Nicolson, colectada en El Nacimiento, muncipio Amatlán de los Reyes, Ver., en vegetación secundaria de selva mediana subcaducifolia.

5. Costus scaber Ruiz \& Pavón, procedente de la Barranca del Coyotito, municipio Tenampa, Ver., en vegetación transicional entre bosque mesófilo de montaña y selva alta perennifolia.

6. Costus pictus D. Don, originaria del ejido Agustín Melgar, municipio Hidalgotitlán, Ver., en selva alta perennifolia.

7. Costus pulverulentus Presl, colectada en la Sierra del Madrigal, municipio Teapa, Tabasco, en selva alta perennifolia.

Los recuentos cromosómicos se efectuaron en un individuo de cada especie mediante el método de aplastado de puntas de raíz y tinción, con la técnica de Feulgen. Las preparaciones permanentes se hicieron con la técnica de "hielo seco" descrita por Sharma y Sharma (1980).

Para determinar el número cromosómico de Costus pictus se realizó un conteo meiótico a partir de botones florales, fijados en solución Carnoy y teñidos con aceto-carmín. Los conteos se realizaron con óptica de contraste de fases con un microscopio Zeiss Fomi III provisto con un objetivo planapocromático (X63). La cámara lúcida se usó como apoyo para determinar el número cromosómico.

\section{RESULTADOS Y DISCUSION}

El cuadro 2 muestra los números cromosómicos de los siete taxa investigados. La contracción extrema de los brazos durante el pretratamiento no permitió estudiar el cariotipo. El conteo haploide de Costus pictus fue $n=9$, que de acuerdo con Sato (1960) es un número básico común dentro de las Zingiberales con cariotipo estable; asimismo el número diploide de $C$. pictus fue $2 n=18$. Costus pulverulentus y $C$. scaber también mostraron $2 n$ =18, lo que está de acuerdo con los conteos realizados por Maas (1972). En México Costus scaber se distribuye en Chiapas, Puebla, Querétaro, centro y sur de Veracruz; en Centroamérica lo hace en Guatemala, Honduras, Costa Rica y Panamá; en América del Sur en Bolivia, Brasil, Colombia, Ecuador, las Guayanas, Perú, Surinám y Venezuela. En las Antillas se conoce de Haití, Granada, Trinidad y Tobago. Costus dirzoi, una especie descrita recientemente y endémica de Veracruz (García-Mendoza e Ibarra-Manríquez, 1991), relacionada con C. pictus, y simpátrica con esta última en la región de los Tuxtlas, mostró $2 n=27$ y 28 , lo que aparentemente representa la condición triploide o triploide +1 . Costus pictus, con $2 n=36$, citado por Venkatasubban (1946), es probablemente tetraploide. Costus pictus se distribuye en México sobre la vertiente del Pacífico desde Sonora hasta Chiapas y en la del Golfo en los estados de Hidalgo, San Luis Potosí y Veracruz. En Centroamérica se conoce de ambas vertientes hasta Costa Rica. Es posible que el género 
Vovides y Lascurain: Números Cromosómicos de Costus, Calathea, Maranta y Stromanthe

Cuadro 2. Número cromosómico de las especies estudiadas.

\begin{tabular}{lcllcc}
\hline ESPECIE & \multicolumn{2}{c}{ REGISTRO PREVIO } & \multicolumn{2}{c}{$\begin{array}{c}\text { ESTE ESTUDIO } \\
(2 n)\end{array}$} \\
& $(2 n)$ & Autor & $(n)$ \\
\hline Calathea ovandensis & -- & & 25 & -- \\
Costus pictus & 36 & Venkatasubban $(1946)$ & 18 & 9 \\
Costus pulverulentus & 18 & Maas (1972) & 18 & -- \\
Costus scaber & 18 & Maas (1972) & 18 & -- \\
Costus dirzoi & -- & & 27,28 & -- \\
Maranta gibba & -- & & 40 & -- \\
Stromanthe macrochlamys & -- & ca. 60, 63 & -- \\
\hline
\end{tabular}

Costus forme una serie poliploide. Costus pictus y $C$. pulverulentus probablemente pueden llegar a formar híbridos naturales en el sur de Veracruz y Tabasco (Maas, 1972; Vovides, 1994), lo que se puede deber a la posible alta fertilidad gamética, ya que ambas especies tienen el número cromosómico diploide de $2 n=18$. Costus pulverulentus es la especie más ampliamente distribuida en la vertiente del Golfo en México y la más variable en cuanto a caracteres vegetativos tales como el indumento y la forma de la hoja. En México se registra de los estados de Chiapas, Oaxaca, Querétaro, Tabasco y Veracruz; en Centroamérica de ambas vertientes; en Sudamérica de Colombia, Ecuador y Venezuela. Algunos ejemplares de $C$. pulverulentus tienen caracteres similares a los de Costus pictus, especialmente el color del cáliz y de las brácteas (Vovides, 1994). Maas (1972) sospecha la existencia de introgresión entre estas dos especies en Tabasco.

Maranta gibba tiene $2 n=40$; se distribuye en regiones cálido húmedas de México, en Campeche, Chiapas, Jalisco, Oaxaca, Puebla, Quintana Roo, San Luis Potosí, Tabasco, Veracruz y Yucatán; en Centroamérica se encuentra desde Guatemala hasta el sur de Nicaragua. Además se conoce de las Antillas menores y en América del Sur de Venezuela, norte de Colombia, Guyana y el oeste de Surinám.

Para Calathea ovandensis el recuento fue de $2 n=25$; esta especie es endémica de México y se distribuye en Chiapas, estado de México, Oaxaca, San Luis Potosí, Sinaloa y Veracruz.

Stromanthe macrochlamys, con $2 n=$ ca. 60 , ca. 63 , se ha colectado en el sureste de México en Chiapas, Oaxaca, Tabasco y Veracruz. En Centroamérica, probablemente en Guatemala.

No existe información suficiente en general sobre la familia Marantaceae para comparar los recuentos presentes y solamente podemos inferir que las especies estudiadas probablemente son parte de una serie poliploide. Para entender mejor la posible poliploidia en este grupo se necesita investigar más material y de otras localidades.

\section{AGRADECIMIENTOS}

Los autores agradecen a F. Chiang, L. Andersson, H. Kennedy y N. Ogata por sus comentarios y consejos. Esta investigación fue realizada gracias al apoyo del Consejo Nacional de Ciencia y Tecnología a través del proyecto CONACyT No. 0063-N9106. 


\section{LITERATURA CITADA}

Andersson, L. 1981. The neotropical genera of Marantaceae. Circumscription and relationships. Nord. J. Bot. 1: 218-245.

Boehm, K. 1931. Embryologische Untersuchungen an Zingiberaceae. Planta 14: 411-440.

Dahlgren, R., H. T. Clifford y P. F. Yeo. 1985. The families of Monocotyledons. Springer-Verlag. Berlin. $520 \mathrm{pp}$.

García-Mendoza, A. y G. Ibarra-Manríquez. 1991. A new species of Costus (Costoideae, Zingiberaceae) from Veracruz, Mexico. Ann. Missouri Bot. Gard. 78: 1081-1084.

Heywood, V. H. 1993. Flowering plants of the world. Oxford University Press, Nueva York. pp. 300301.

Kress, W. J. 1990. The phylogeny and classification of the Zingiberales. Ann. Missouri Bot. Gard. 77: 698-721.

Maas, P. J. M. 1972. Costoideae (Zingiberaceae). Flora Neotropica, Monograph No. 8. New York Botanical Garden, Nueva York. $139 \mathrm{pp}$.

Mahanty, H. K. 1970. A cytological study of the Zingiberales with special reference to their taxonomy. Cytologia 35: 13-49.

Moore, R. J. (ed.) 1973. Index to plant chromosome numbers 1967-1971. Regnum Veg. 90: 1-530.

Raven, P. H. 1975. The bases of angiosperm phylogeny: cytology. Ann. Missouri Bot. Gard. 62: 724764.

Sato, D. 1948. The karyotypes and phylogeny in Zingiberales. Jap. J. Genet. 23: 44-45.

Sato, D. 1960. The karyotype analysis in Zingiberales with special reference to the protokaryotype and stable karyotype. Sci. Pap. Coll. Gen. Educ. Univ. Tokyo 10: 225-273.

Sharma, A. K. y Sharma, A. 1980. Chromosome techniques, theory and practice. 3a. ed. Butterworths, Londres. $711 \mathrm{pp}$.

Venkatasubban, K. R. 1946. A preliminary survey of chromosome numbers in Scitamineae of Bentham and Hooker. Proc. Indian Acad. Sci. 23B: 281-300.

Vovides, A. P. 1994. Costaceae. Flora de Veracruz. Fascículo 78. Instituto de Ecología, A.C., Xalapa, Ver. $13 \mathrm{pp}$.

Vovides, A. P. 1993. Familia Zingiberaceae. Flora del Bajío y de Regiones Adyacentes, Fascículo 18. Instituto de Ecología, A.C. Pátzcuaro, Mich. 14 pp. 